\title{
How do You Feel in Virtual Environments? The Role of Emotions and Openness Trait Over Creative Performance
}

\author{
Sergio Agnoli 1,2 , Sofia Zenari3, \\ Serena Mastria1,2, and Giovanni Emanuele Corazza1,2,4
}

\author{
1 Marconi Institute for Creativity (MIC), Villa Griffone, Sasso Marconi, Bologna, Italy \\ 2 Department of Electrical, Electronic, and Information Engineering "Guglielmo Marconi", University of Bologna, Bologna, Italy \\ 3 Department of Architecture, University of Bologna, Bologna, Italy \\ 4 Université de Paris and Univ Gustave Eiffel, LaPEA, Boulogne-Billancourt, France
}

\section{ABSTRACT}

In the Dynamic Creativity Framework creativity is defined as a context-embedded phenomenon requiring potential originality and effectiveness. This definition indicates that the environmental conditions embedding the creative process have fundamental impact on the process itself and its outcomes. In particular, Virtual environments (VEs) are emerging as everyday contexts for a large part of the world population, affecting behaviors and feelings. VEs have been demonstrated to affect creative performance in several ways, even if the psychological mechanisms at the basis of the different modifications in the creative behavior are far from being completely explained. The aim of this study was to explore the influence of different types of VEs on creative performance, with a specific focus on participants' emotional reactions and on their individual differences in the Openness personality trait. A total of 22 participants were exposed to four different types of environments: a real room environment (RE), a control virtual environment (CVE) resembling the physical characteristics of the $\mathrm{RE}$, a positive virtual environment (PVE) and a negative virtual environment (NVE). Participants were free to explore each environment for two minutes, then they were
\end{abstract}

asked to perform an Alternative Uses Task for five minutes, to measure divergent thinking performance. Openness and affective reactions in each environment were measured in all participants. Results showed that Openness was associated with higher originality of responses and that this effect was particularly significant in PVE. Importantly, the type of environment interacted significantly with participants' affective reactions in explaining their creative performance, revealing that an increase of ideas originality was associated with an increase of positive affect, emerging as a consequence of experiencing a PVE. Affective reactions to VEs, in combination with individual differences in term of Openness, thus emerge as one of the possible explicatory mechanisms of the impact of virtual reality on creative performance.

\section{KEYWORDS:}

virtual environment, divergent thinking, emotions, openness, creativity

\section{Article history:}

Received: May 31, 2021

Received in revised from: June 28, 2021

Accepted: July 2, 2021

ISSN 2354-0036

DOI: 10.2478/ctra-2021-0010

\section{Corresponding author at:}

Sergio Agnoli

E-MAIL: sergio.agnoli@unibo.it 


\section{INTRODUCTION}

In the Dynamic Creativity Framework (DCF), creativity is defined as a "context-embedded phenomenon requiring potential originality and effectiveness" (Corazza \& Lubart, 2021). This definition, which is an evolution of the dynamic definition of creativity (Corazza, 2016), indicates clearly that the environmental conditions embedding the creative process have fundamental impact on the process itself and its outcomes. While in the past the 'environment' alluded to the actual physical conditions around an individual, over the past 30 years, information and communication technologies have transformed the way in which we communicate, acquire knowledge, share experiences, and, substantially, live by introducing Virtual Environments (VE). New possibilities and degrees of freedom have thus been introduced in our lives, creating new contexts where to express our behavior. These new technologies introduce new challenges for the human beings, who must be able to adapt in order to exploit at their best the possibilities offered by the new contexts. VEs are in particular able to connect people who are distant in space and in time, allowing new opportunities for supporting and fostering creativity (Guegan et al., 2016; Thornhill-Miller et al., 2016). From a scientific point of view, it is particularly important understanding which are the main mechanisms through which these environments can influence the human creative behavior. VEs are indeed emerging as new promising opportunities both to increase the individual and the team creative potential (Bourgeois-Bougrine et al., 2019; Guegan et al., 2017; Michinov et al., 2015 ) and to understand the key role of specific cognitive, emotional, and personality factors on the creative performance (Bourgeois-Bougrine et al., 2019; Guegan et al., 2016; Pena et al., 2009). Within the DCF, the current work specifically aimed at understanding the role of emotional and personality mechanisms as possible explanatory mechanisms of the influence of the exposure to VEs on creative performance, in line with the dynamic definition of creativity.

Psychological research has extensively explored how the environment can influence the human behavior, and recently increasing attention has been also devoted to the understanding of the VE characteristics influencing thoughts and behaviors in several life contexts (see Guegan et al., 2017). From an experimental point of view, virtual reality offers the promising possibility of developing extremely controlled environments as well as of specifically manipulating experimental variables, which could be hardly manipulable in the real world. VEs are indeed computergenerated environments that allow the user to interact with the virtual space and objects using interactive items (i.e., avatars) through a display screen (Vergara et al., 2015). Several studies have thus explored the environmental cues and the avatars' features influencing creative performance both of single users and of teams (Bourgeois-Bougrine et al., 2019; Guegan et al., 2016; Pena et al., 2009). Research showed for instance that fluency and idea uniqueness in creativity tasks as well as users' contents of narrative productions can be modulated through the manipulation of avatars' appearance (Guegan et al., 2016; Pena et al., 2009). Engineering students embodying an "inventor" avatar emerged indeed to generate more original contents than students embodying a "non-inventor" neutral avatar (Guegan et al., 2016). Beyond avatars' appearance, the design of 
the whole VE can influence creative performance. Guegan et al. (2017) indeed showed that the exposure to a creativity-conducive virtual environment was able to conduct to more original and elaborated ideas in comparison to a virtual control environment and a real control environment. They specifically demonstrated that the increase in creative performance was related to the nature of the contextual cues that were present in the environment rather than to the technological medium (virtual vs. real) through which they were presented.

Fostering creative potential through the use of VEs requires however, as suggested by Guegan et al. (2017), a better knowledge of the mechanisms through which VEs can influence creative behavior. Several explanatory mechanisms have been proposed, including the influence of VEs on users' cognitive mechanisms (e.g., Kalantari et al., 2021; Ragan et al., 2012), as well as on their affective states and mood (Chirico et al., 2016; Chirico \& Gaggioli, 2019; Shin, 2018). This latter type of mechanism appears to be particularly intriguing, considering the vast amount of literature demonstrating the influence of emotions on creative performance (see for a meta-analysis, Baas et al., 2008). Research in particular showed that positive affective states, in comparison to neutral conditions, are able to increase divergent thinking (i.e., fluency, originality, flexibility) and insight problem-solving tasks performance, since individuals tend to make richer associations between information during a positive affective state than when in a neutral state (Ashby et al., 1999; Forgas, 2000; Isen et al., 1987). Contradictory results emerged instead on negative affective states, with some studies showing no differences in creative performance when individuals are in a negative affective state than in a neutral condition, whereas other studies showed a slightly detrimental effect of the negative states on creativity (Goritz \& Moser, 2003; Verhaeghen et al., 2005). According to Bourgeois-Bougrine et al. (2019) VEs might offer a "liberating" atmosphere to the users that is able to disinhibit the use of more affective laden risky behaviors, which lead to higher creative performance. Emotions can therefore be a mechanism to produce more creative behaviors in VEs. Moreover, according to these authors, this disinhibited atmosphere could interact with users' attitudes and in particular with their Openness personality trait, leading to the production of more remote and original contents. Openness has indeed been showed to be one of the main predictors of creative performance and achievement (Agnoli et al., 2015; Corazza \& Agnoli, 2020; Feist, 1998; Jauk et al., 2014). They thus hypothesized that the Openness trait can be involved as explicatory mechanism in the influence of VEs on the creative behavior.

Therefore, whereas it is clear that the environmental cues characterizing VEs as well as avatars' characteristics are able to influence users' creative performance, it is still not clear whether the users' affective states emerging during the exposition to these environments can affect their creative behavior. Moreover, it would be worth exploring whether the VEs characteristics can interact with the users' personality traits, and in particular with the Openness trait, in predicting creative performance. 


\section{THE CURRENT STUDY: AIMS AND HYPOTHESIS}

In the present exploratory study, we offered a first examination of the above mentioned research questions. We specifically designed two different types of VEs, i.e., a VE characterized by positive (PVE) cues and a VE characterized by negative (NVE) cues, in order to explore their influence on users' affective states and on the divergent thinking (DT) performance. DT performance as well as participants' affective states associated to these two types of environments have been compared to a neural real environment (RE) and to a control virtual environment (CVE), which totally resembled the physical features of the real environment. Since avatar's appearance is able to influence creative behavior (Guegan et al., 2016; Pena et al., 2009), we used neutral avatars that could not be personalized by the users. Moreover, we measured individual differences in the Openness personality trait, in order to explore their interactions with the different environments in affecting the participants' creative performance.

We specifically hypothesized that:

1) The positive and the negative $V E$ could lead to an increase of positive and negative affective states, respectively, in comparison to the control conditions.

2) The positive affective reactions emerging during the interaction with the PVE could predict a higher DT performance in comparison to the neutral (both real and virtual) conditions and to the negative conditions (NVE). The negative affective states emerging from the interactions with the NVE should not instead produce any change in participants' creative performance, or only a slight decrease of the creative performance.

3) The Openness personality trait might interact with the environment characterized by the more "liberating" atmosphere (i.e., PVE) in increasing the DT performance.

\section{METHOD}

\section{Participants}

A total of 22 participants were involved in the study ( 7 females, mean age $=22.86, S D=1.42$; age range $=19-25$ ). All participants were informed they will be involved in a study exploring creativity in real and virtual environments. Participants had no previous experience with the materials used in this experiment. They all voluntarily participated in the study and signed a written consent before starting the experiment. The present research conformed to the Declaration of Helsinki and participants were free to participate in or abstain at any time from the research. We a-priori calculated the required sample size to achieve $80 \%$ statistical power to detect an adequate effect by G*power 3.1 (Faul et al., 2007) software. The power calculation was based on the effects obtained in previous research on the influence of virtual environment on creative performance (BourgeoisBougrine et al., 2019), which reported a small to medium effect size, and yielded a recommended sample size of 17 participants. 


\section{Instruments}

\section{Emotional states: PANAS-X (Watson \& Clark, 1999)}

PANAS-X is a 60-item consisting of a number of words that describe different feelings. Participants were asked to rate the intensity of the feelings they experienced on a 5-point scale ranging from 1 (very slightly) to 5 (extremely). For the purpose of this study, we used only the 20 items used to compute the two general dimensions scales: Positive affect (10 items) and Negative Affect (10 items). The reliability of the two scales was good ( $a>0.71)$.

\section{Openness: NEO Five Factor Inventory (NEO-FFI; Costa \& McCrae, 1992)}

NEO-FFI assesses the five personality traits as theorized by the Big- 5 model (Costa \& McCrae, 1992). It consists of 60 items providing a measure of Neuroticism, Extraversion, Openness to Experience, Agreeableness, and Conscientiousness. Participants were required to answer to sentences (e.g., "Sometimes when I am reading poetry or looking at a work of art, I feel a chill or wave of excitement", as an Openness item) reporting their agreement/disagreement level with the sentence on a 5 -point scale ( 1 = strongly disagree, $5=$ strongly agree). Here for the purpose of the study, only the 9 Openness items of the NEO-FFI inventory were used to obtain participants' Openness scores (scale reliability, $a=0.91$ ). A total Openness score was obtained by summing the Openness items after the items recoding performed in accordance with the NEO-FFI scoring manual.

\section{Virtual environments: Sansar Platform}

Three specific virtual environments have been created on the Sansar platform (https:// www.sansar.com), allowing the use of an advanced graphics to generate new ad hoc 3D virtual environments that can be freely explored using avatars, which can be in turn developed within this platform. Specifically, two environments have been generated as characterized by opposite characteristics, a first one based on open, bright, and comfortable elements, the second one based on close, dark, uncomfortable elements. On the basis of past research on the use of virtual environmental cues to induce affective states (e.g., Felnhofer et al., 2015; Riva et al., 2007), the first virtual environment (i.e., positive virtual environment, PVE) has been generated in order to elicit positive attitudes in participants through the use of positive cues (see Figure 1). Specifically, a tropic island has been chosen as the main background with wide spaces for exploration. The horizon is always visible to the user, and its view is quite never hampered by obstacles. Sea and sand beaches are present in this environment as well as low mountains. The environment is enriched by inflatable balls and mats, deck chairs, games for children, benches and areas to sit or linger, palm trees and other types of trees, a landing stage, a floating platform and more. The lighting is very bright recalling the sunlight during the midday. 

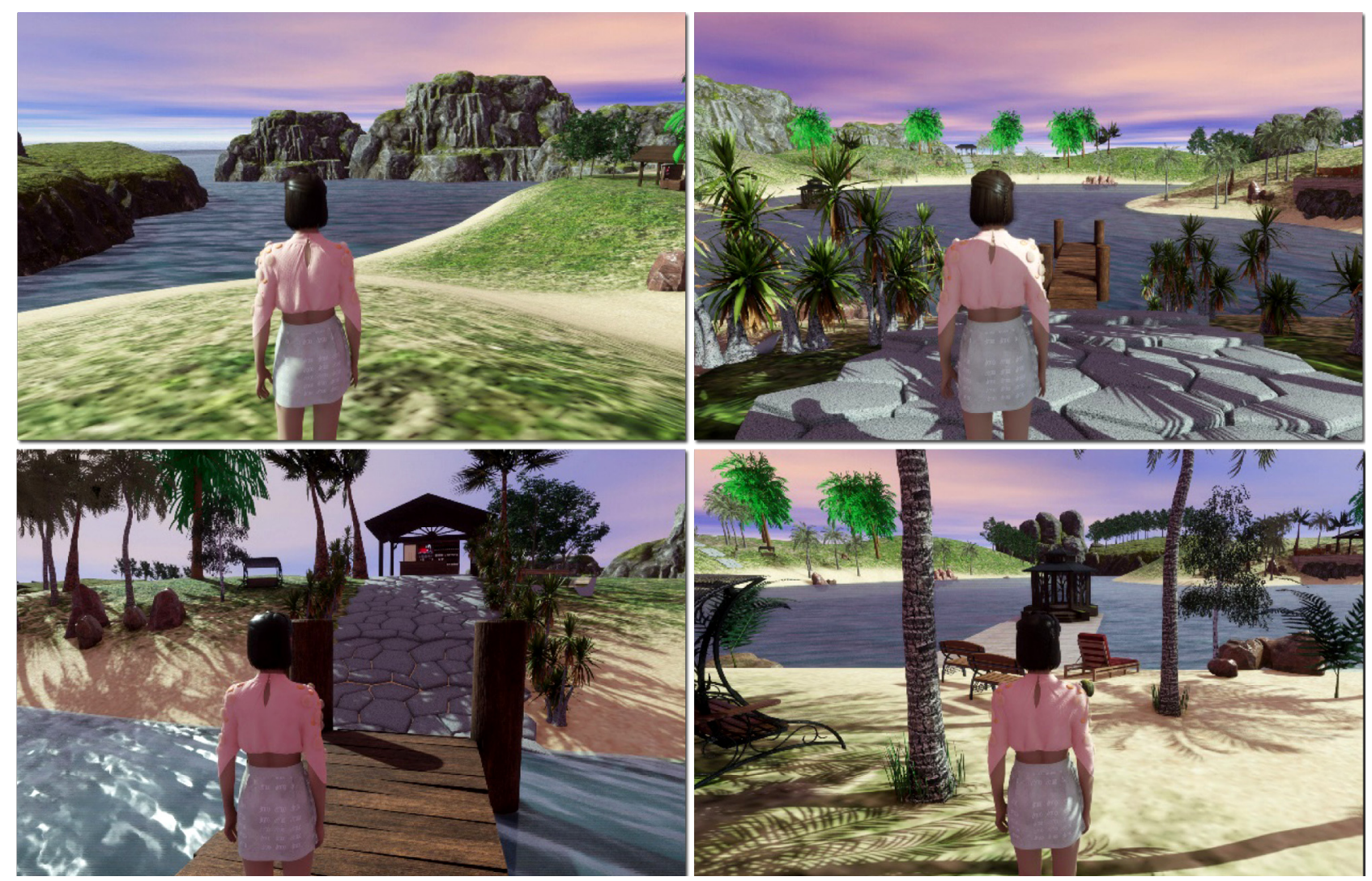

Figure 1. Examples of scenarios representing the positive virtual environment

The second virtual environment (i.e., negative virtual environment, NVE) has been instead generated to elicit negative attitudes in participants through the use of negative cues (see Figure 2). It is based on a rectangular surface. On each side of the surface a barrier has been included, in order to perfectly close this space and generate a retaining wall. In the center of the space, a room is located, which is almost completely unadorned and dark, containing only few elements (a table, a chair and a bed) and recalling the cell of an abandoned prison. This room is surrounded by thick walls with an opening, which allows the entrance and the exit. The atmosphere of this environment is very dark with a perception to be in a scary and abandoned place. The sky is completely dark, but there is a spot of intense light that, coming into contact with the elements of the environment, creates sharp and defined shadows. Several elements are included in this scenario: spiders, bare trees, withered leaves.

The third virtual environment (i.e., control virtual environment, CVE) have been generated in order to resemble the physical characteristics of the real environment where participants were physically located (see Figure 3). The real environment (RE) was a room of a house, which have been left as unadorned as possible, removing any unnecessary furnishings in order to not include perceptual distractors for the participants. The CVE resembles so far as possible the physical features of the real room. 

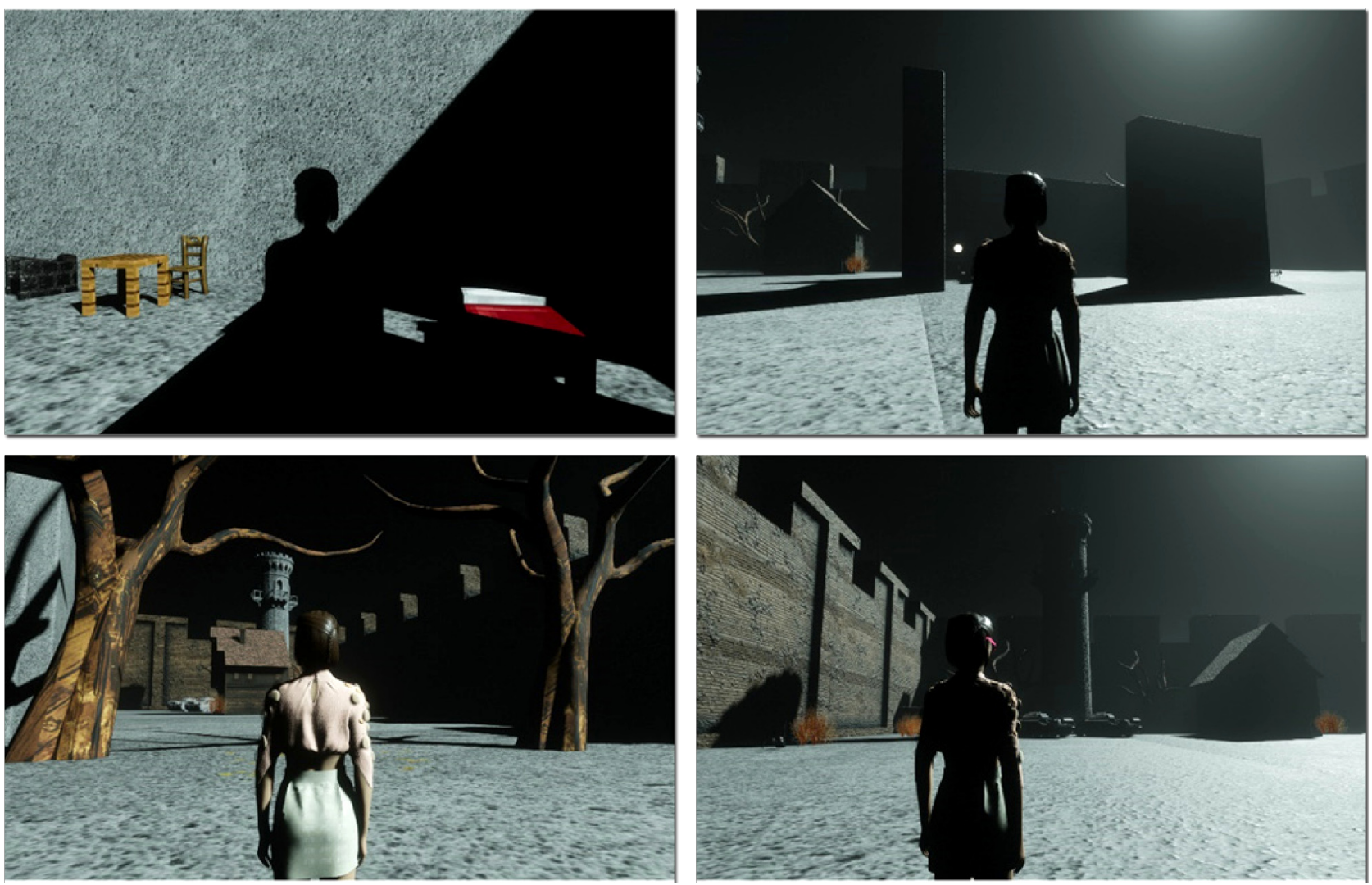

Figure 2. Examples of scenarios representing the negative virtual environment

Real environment
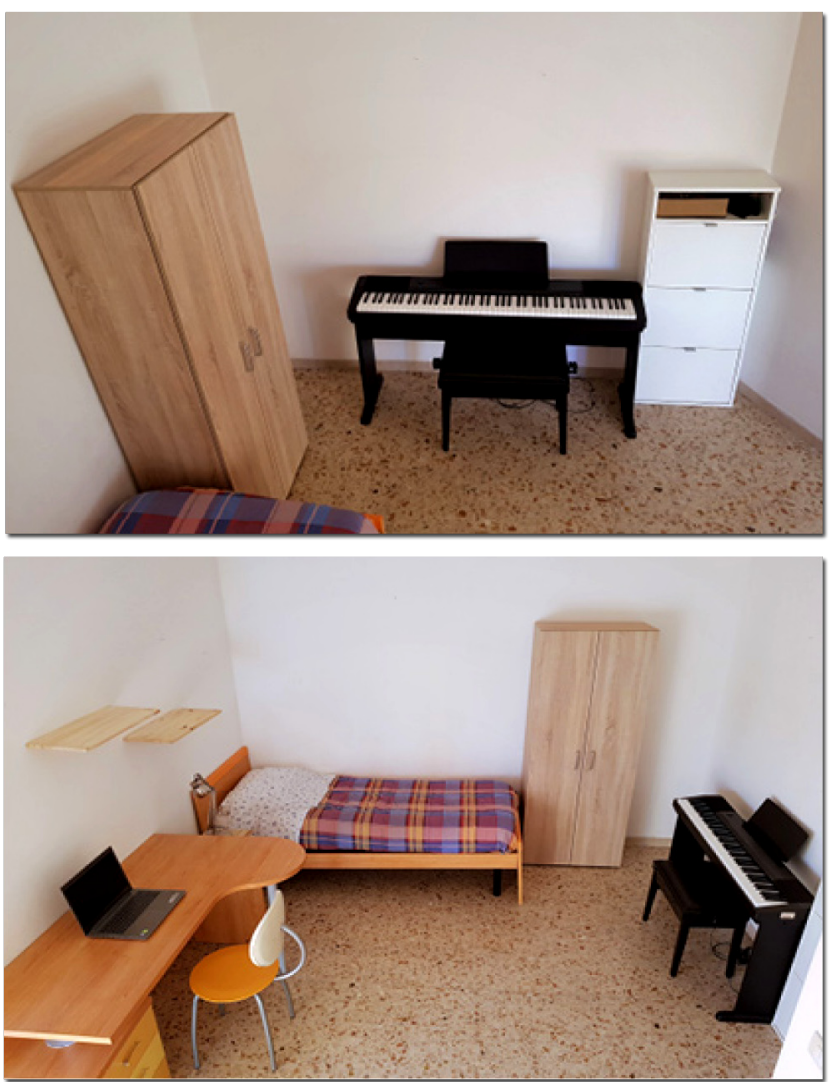

Virtual environment
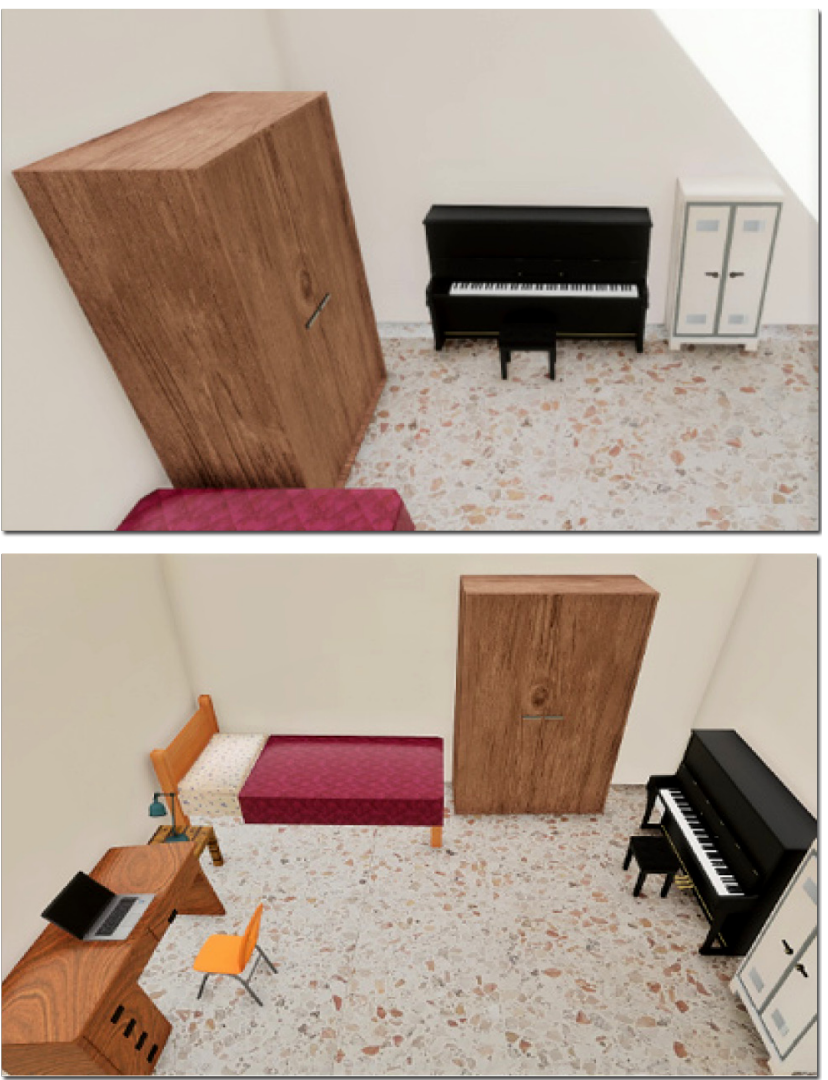

Figure 3. Two perspective views comparing the real environment (a real room, on the left) and the control virtual environment (on the right) 


\section{PROCEDURE}

Before the beginning of the experiment, participants were asked to answer a paper and pencil version of the PANAS- X questionnaire (Watson \& Clark, 1999). Specifically, participants were asked to rate the intensity of their feelings according to "how they felt at that present moment" (Watson et al, 1988). Participants were then told that they would participate in a computer task that they had to perform different times using diverse stimuli in different environments, some of which were virtual and one of which was real.

The order of the four environments (PVE, NVE, CVE, RE) was counterbalanced across participants. At the entrance in each environment, participants were instructed that they can freely explore the environment for two minutes (this time window for the familiarization period was chosen because of the within-nature of the study, in order to limit the time of the experiment per each participant). Virtual environments were presented through a laptop computer (with a 15.4-inch monitor), which was used also for the AUT in the real environment. In the real environment participants could physically walk through the space of the room. In the virtual environments, they were said they could move through the environment using the four arrow keys on the laptop keyboard. All virtual environments were tested in a different physical space than the real environment. Entering in the first virtual environment a stereotypical avatar was assigned to the participants (a male avatar to male participants, a female avatar to female participants). Moreover, they were instructed that they could move the framing (avatar's perspective) using the right button of a mouse connected to the computer. At the end of the second minute, participants were asked to participate in an AUT task, requiring to produce original alternative uses for one common object (word stimuli) according to the following instructions "Here you can find the word of an object, for which you are asked to produce as many alternative uses as you can. For example, for the object "hat" an alternative use could be "jewel box". There are not right or wrong answers, so please feel free to write any alternative use it comes into your mind. Try to have fun and to be original. You have 5 minutes of time for writing your original alternative uses". Four different objects were used (tin can, carton box, glass bottle, car tyre) in the four environments (one per environment), whose order was again counterbalanced across environments and across participants (see Figure 4).

In the virtual environments, the instructions and the word of the object appeared at the end of the exploration phase (after 2 minutes) on a chat window on the right part of the monitor, where participants were asked to write their responses. In the real environment, at the end of the exploration phase, the instructions and the object appeared on a text sheet on the laptop screen placed on a desk at the centre of the room (see Figure 3). Participants were then asked to sit at the desk and to write their responses on the laptop. At the end of the AUT task, in each environment participants were asked to answer to a paper and pencil version of the PANAS-X questionnaire, again asking to rate the intensity of their feelings at present moment. Finally, at the end of the experiment, participants were asked to answer to the NEO-FFI questionnaire. The entire experimental procedure had a total duration of about 50 minutes. 


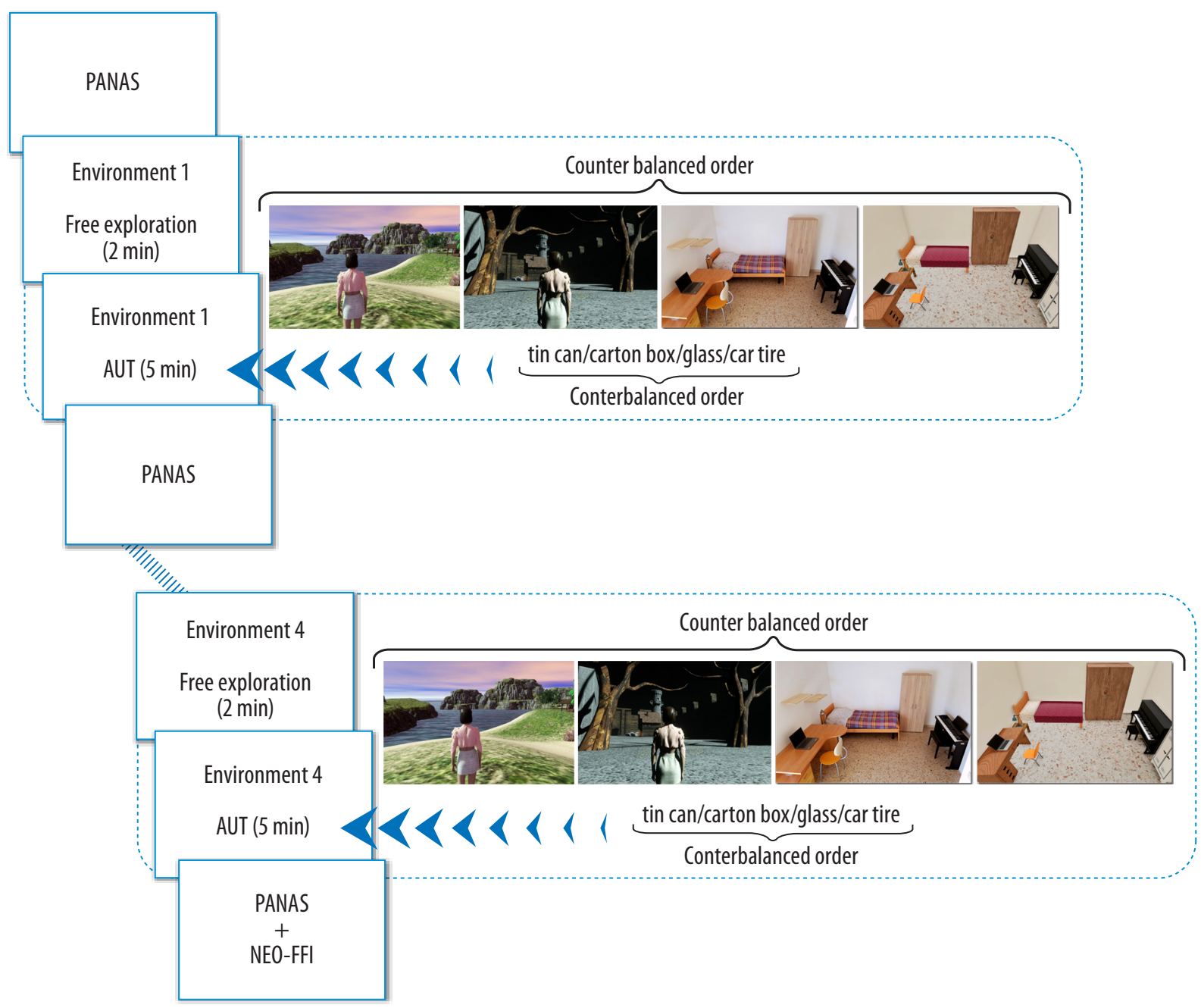

Figure 4. Experimental procedure. Before the beginning of the experiment participants completed the PANAS questionnaire. Each participant was then introduced in four different environments (three virtual environment - positive, negative, control - and a real environment), whose presentation order was counterbalanced across participants. They could freely explore each environment for 2 minutes and then they were involved in an AUT task for 5 minutes (producing original alternative use for one of four common objects). At the end of the task in each environment, they were required to complete again the PANAS questionnaire and at the end of the experimental procedure the NEO-FFI questionnaire

\section{Assessment of DT performance}

At the end of the data collection two independent expert raters judged participants responses for originality. Responses were previously transcribed into a spreadsheet and then sorted alphabetically within each target object. In this way, the raters were blind to the response serial position in the set, the objects order, the total number of responses in the set, the participant who produced the response, and the preceding and following responses. The raters first read all responses for an object, then scored each response individually. Consistently with previous studies (Wilson et al., 1953; Silvia et al., 2008), each response received a rating on a 1 (not at all original) to 5 (highly original) scale. The inter-rater reliability calculated on the total number of alternative uses was acceptable (Cohen's 
$\mathrm{K}=0.605$ ). Finally, the two ratings were averaged into one originality score for each idea. Besides expert external raters' originality scores also the number of responses (i.e., fluency) produced by each participant in the different environments was calculated.

\section{DATA ANALYSIS}

A series of Generalized Linear Mixed Models (GLMM) was performed in order to test our hypotheses, controlling for the random effect of subjects and using a robust error estimation in order to control for the effect of outliers ( $\mathrm{Wu}, 2009)$. Where necessary, Bonferroni corrected post-hoc comparisons were used to further explore differences between the levels of the explored variables.

\section{RESULTS}

\section{Positive and negative affect}

First, the effects of the different environments (PVE, NVE, CVE, RE) on participants' positive and negative emotional states were explored. Specifically, the positive and negative affective values recorded at the end of each environment were subtracted from the values recorded at the beginning of the experiment (baseline). Changes in positive and negative affects due to the different environments were therefore taken into account in comparison to a baseline state: positive values mean an increase in positive or negative affect, negative values indicate a decrease in the two type of affect. It is worth highlighting that the negative affect values in the baseline condition showed a moderate level of negative affect probably due to uncertainty and anxiety linked to the experimental situation (see Table 1). A first GLMM model explored the effect of the environment (4 levels: PVE, NVE, CVE, RE) on the changes of positive affect, as dependent variable. Results showed that the type of environment has a significant effect on the change of positive affect in participants, $F(3,84)=6.03, p=.001$. In particular, as shown in Figure 5A, PVE induced a higher increase in positive affects in comparison to the other environments. Specifically, a higher increase in positive affect in PVE emerged as compared to the NVE, $b=0.209, t(84)=3.36, p=.001,95 \% \mathrm{Cl}[.085, .333]$, and the $\mathrm{CVE}, b=0.209, t(84)=3.10, p=.003,95 \% \mathrm{Cl}[.075, .343]$.

A second model explored the changes of negative affect in relation to the four environments. Again, results showed that the type of environment has a significant effect on the changes of negative affect in participants, $F(3,84)=5.26, p=.001$. Specifically, as shown in Figure $5 B$, the NVE induced a lower decrease in negative affect than in the other environments. In particular, a significant difference in the change of negative affect emerged between NVE and RE, $b=0.314$, $t(84)=3.29, p=.001,95 \% \mathrm{Cl}[.124, .503]$. 

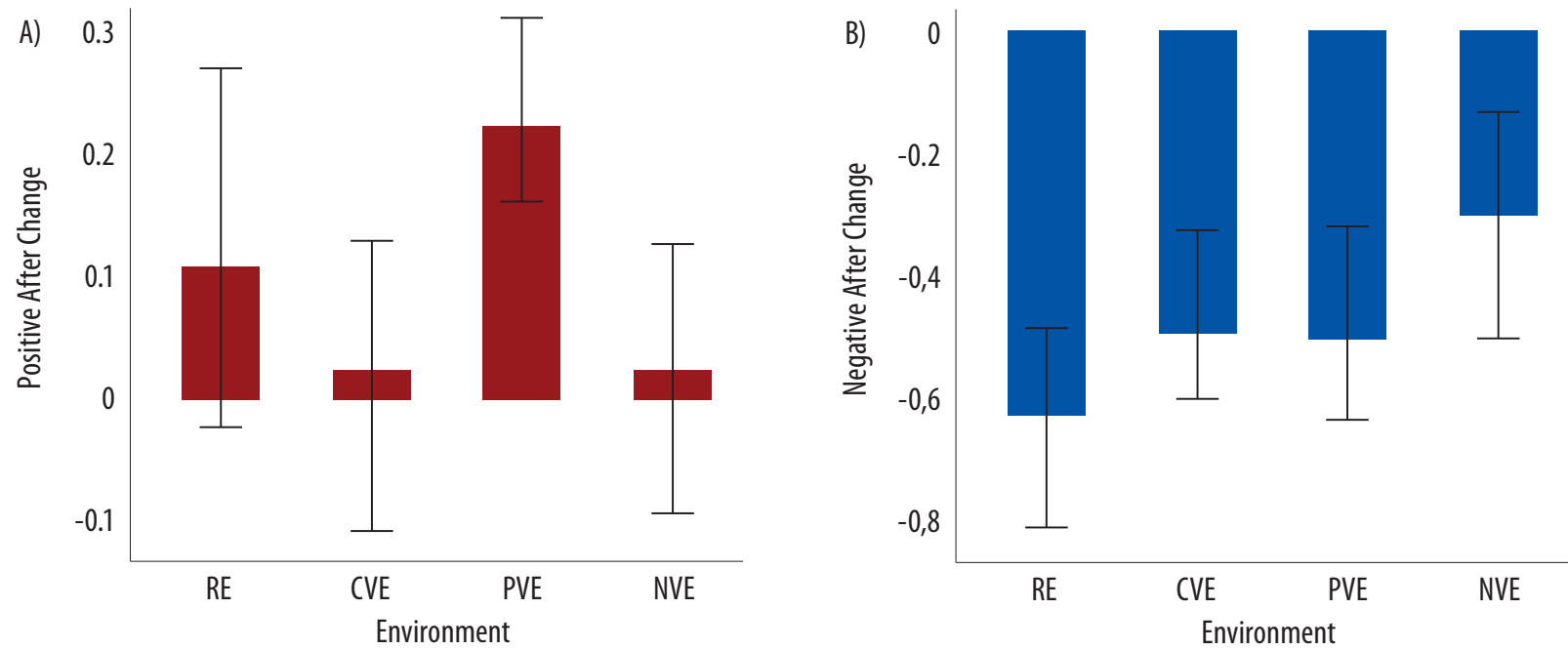

Figure 5. Changes of positive affect (panel A) and negative affect (panel B) as a function of the Real Environment (RE), Control Virtual Environment (CVE), Positive Virtual Environment (PVE), and Negative Virtual Environment (NVE). Lines within the bars represent $95 \%$ confidence intervals (Cl)

\section{Creative performance and affective states}

A second series of analyses explored the role of the change in the affective states and of the different environments, as well as of their interaction, on the creative performance, i.e., on the originality and fluency of participants' responses (see Table 1). Separate models have been performed for positive and negative affect and for the two dependent variables, originality and fluency.

Table 1. Means and standard deviations (within brackets) of the positive and negative affect values and of the AUT measures in the different experimental conditions

\begin{tabular}{cccccc} 
& Baseline & $\begin{array}{c}\text { Positive Virtual } \\
\text { Environment }\end{array}$ & $\begin{array}{c}\text { Negative Virtual } \\
\text { Environment }\end{array}$ & $\begin{array}{c}\text { Control Virtual } \\
\text { Environment }\end{array}$ & $\begin{array}{c}\text { Real } \\
\text { Environment }\end{array}$ \\
\hline Positive Affect & $3.09(.59)$ & $3.32(.46)$ & $3.11(.53)$ & $3.11(.45)$ & $3.20(.52)$ \\
\hline Negative Affect & $2.10(.87)$ & $1.61(.66)$ & $1.78(.78)$ & $1.63(.75)$ & $1.47(.61)$ \\
\hline AUT Originality & $/$ & $2.36(.41)$ & $2.32(.35)$ & $2.30(.45)$ & $2.42(.36)$ \\
\hline AUT Fluency & $/$ & $8.27(3.22)$ & $8.86(3.87)$ & $8.59(2.95)$ & $9.59(3.81)$ \\
\hline
\end{tabular}

A first model explored the effect of the changes of positive affect in the different environments on originality, showing a significant interaction between the environment condition and the changes in positive affect, $F(3,80)=2.94, p=.038$. Specifically, as shown in Figure 6 , the increase in positive affect in the positive virtual environment was able to predict an increase in the originality of participants responses in comparison to the other environments, and in particular of the CVE, $b=0.473, t(80)=2.30, p=.024,95 \% \mathrm{Cl}[.065, .881]$. The second model testing the effect of the changes in positive affect in the different environments on fluency did not show any significant main or interaction effect, $p s>.097$. 


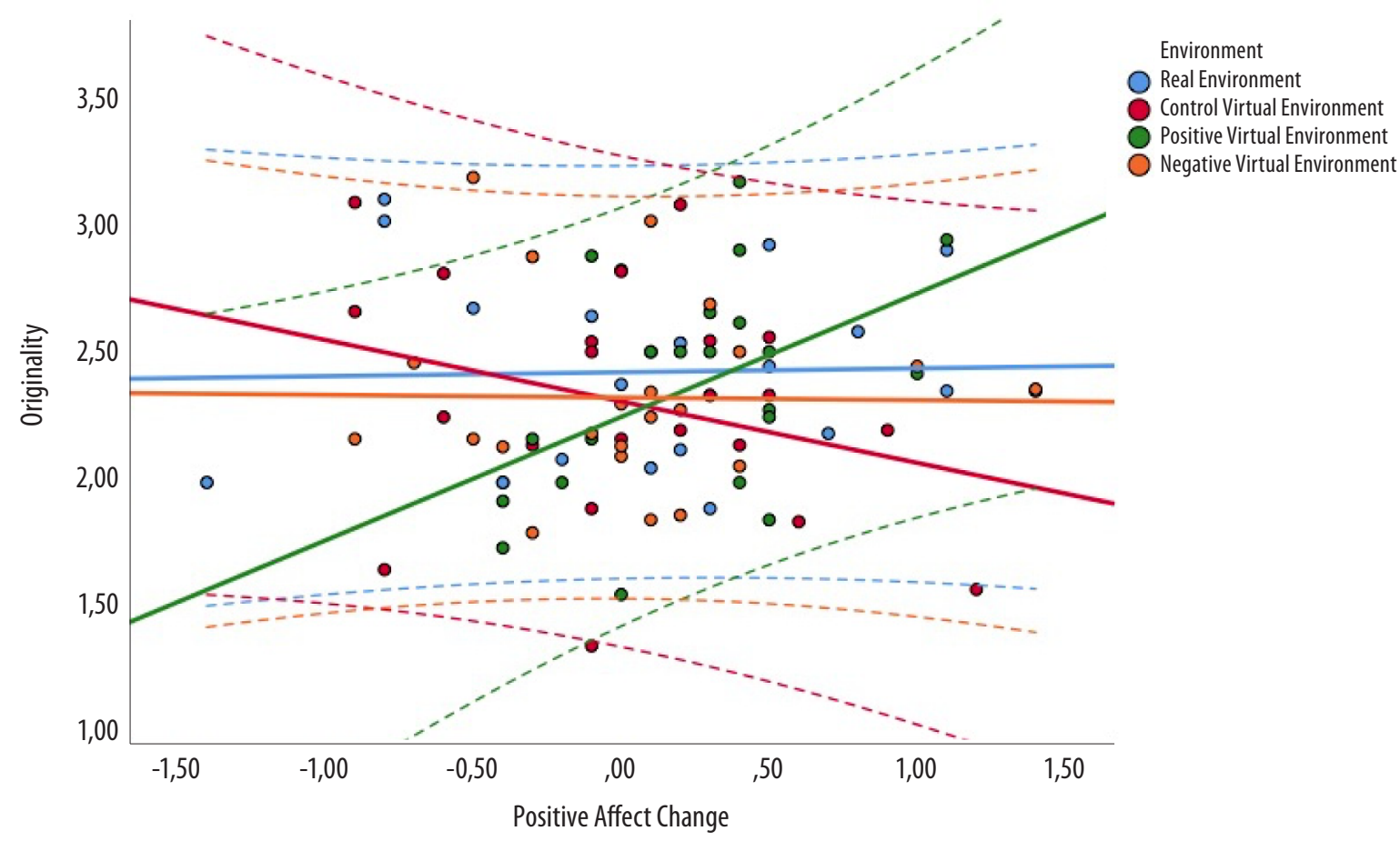

Figure 6. Changes of response originality as a function of the changes in positive affect in the different environments. Dotted lines represent $95 \% \mathrm{Cl}$

A third model exploring the effect of the changes of negative effects in the different environments on response originality did not showed any significant effect, $p s>.077$. The same trend emerged also in the last model predicting fluency through the changes of negative effects in the different environments, where no significant effects emerged, $p s>.34$.

\section{Creative performance and Openness}

A third series of analyses tested our third hypotheses, i.e., whether the different environments can differently interact with the Openness trait to predict creative performance. Specifically, separate models were run on originality and fluency, including Openness and the type of environment as well as their interaction as independent variables. A first GLMM model showed a significant main effect of the Openness trait on response originality, $F(1,80)=5.15, p=.026$, revealing a generalized effect of Openness in predicting the originality of participants' responses across all environments. However, a significant interaction between Openness and the type of environment further specified this main effect, $F(3,80)=3.48, p=.020$. Specifically, as shown in Figure 7 , the PVE emphasized the association between Openness and originality, which emerged to be higher in this environment that in the other environments, and especially than in the CVE, $b=0.039, t(80)=2.14, p=.035$, $95 \% \mathrm{Cl}[.003, .076]$. A final model did not show instead any significant effect on response fluency, ps $>.154$. 


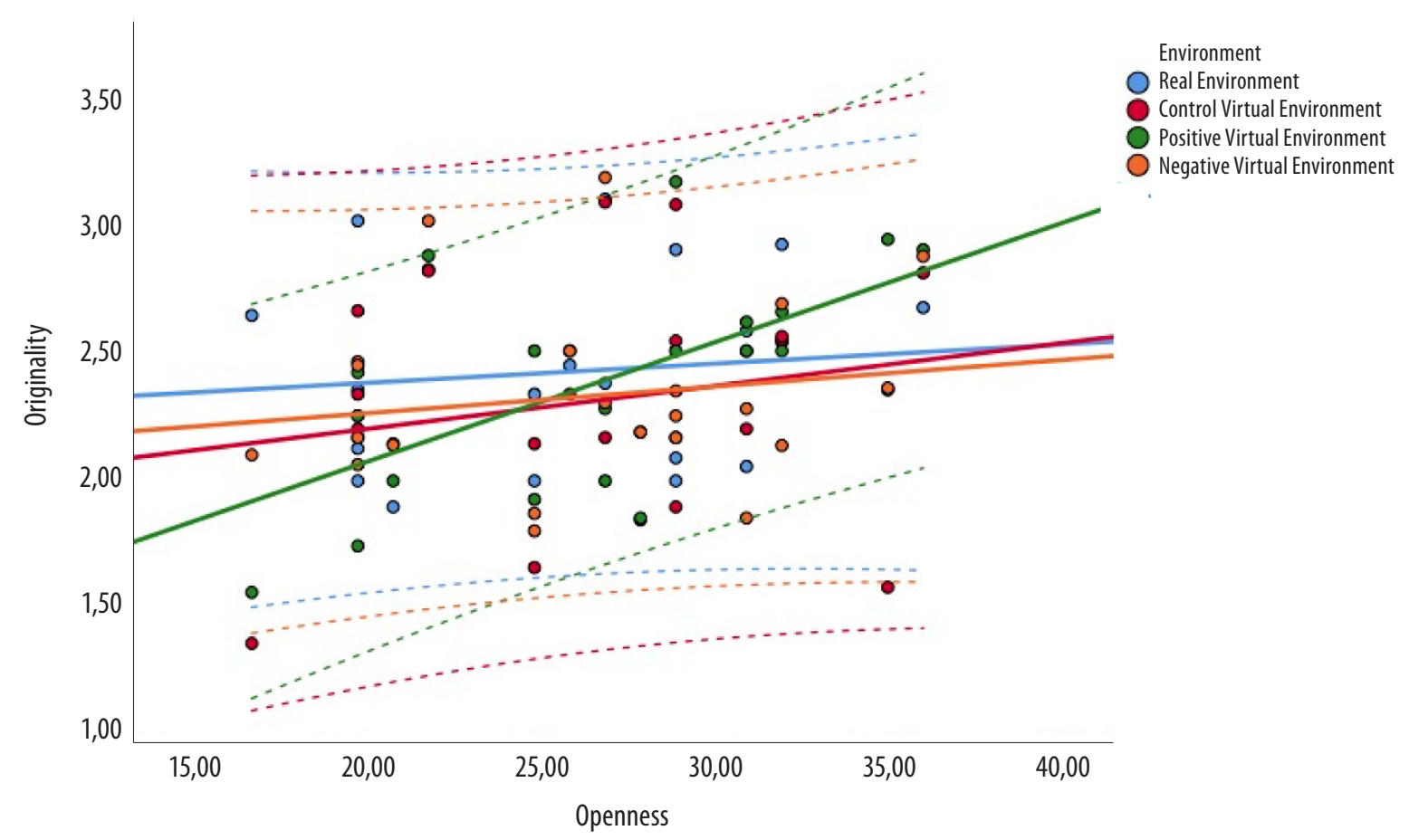

Figure 7. Changes of response originality as a function of Openness in the different environments. Dotted lines represent $95 \% \mathrm{Cl}$

\section{DISCUSSION}

The present exploratory study aimed at understanding whether the affective reactions emerging during the exposure to VEs could be used as possible explanatory mechanisms of the influence of VEs on the creative behavior, in line with the dynamic definition of creativity as a contextembedded phenomenon requiring potential originality and effectiveness. Moreover, we were interested in exploring whether the Openness personality trait could interact with the different VEs in predicting DT performance. For these reasons, two different environments have been designed as characterized by positive cues (PVE) and by negative cues (NVE). The creative performance in these environments was contrasted with the performance in two control conditions, i.e., in a real environment (RE) and in a control virtual environment (CVE) that totally resembles the characteristics of the real condition.

The first result emerging from this study revealed that the different VEs were able to elicit a change in participants' affective reactions, with the PVE inducing an increase in positive affect and the NVE reducing the decrement of negative affect in comparison to the control conditions. This result testifies that the two environments designed on the basis of positive and negative cues were effective in eliciting diverse (and partially opposing) affective reactions. It is worth highlighting the particular effect emerging in the NVE, which did not induce an increase in the negative affective 
states, but a decrease of the reduction of negative affective states. Before the beginning of the experiment participants showed indeed moderate levels of negative affect, probably because of a sense of uncertainty and anxiety for the experimental situation they were going to be involved in. This result is a recurrent effect emerging in the subjective measurement of the negative affective states before the beginning of an experimental study (see for example Agnoli et al., 2015). However, while in the positive and in the control conditions the negative affective states decreased significantly, they did not decrease in the NVE, where they continued to remain at the levels recorded during the baseline. In general, our findings on the modulation of affective reactions by VEs are totally in line with the consistent effect showed by past research on the possibility of inducing and manipulating emotional reactions using virtual reality (Chirico et al., 2016; Chirico \& Gaggioli, 2019; Shin, 2018). VEs could be therefore considered as vehicles for emotional reactions, which are in turn strong determinants of human behavior.

Through the design of environments based on positive and negative cues, we were thus able to induce different emotional experiences in our participants. But could these VEs-emotional experiences influence participants' creative performance? Our results showed that they actually could predict DT performance and in particular the originality of the ideas generated within these environments. Specifically, we found that the increase of positive affective reactions as induced by the experience of the positive virtual environment were able to increase ideas originality. The positive virtual environment was therefore conductive to higher originality responses through the increase of positive affective reactions, whereas this effect did not emerge in the other environments (both virtual and real), which were not able to induce a significant increase in the positive affect. Contrarily, the changes in negative affect did not emerge to be able to influence participants' creative performance. Again, however, it is worth highlighting that our manipulation through the use of NVE was not able to induce an increase of negative affect, which is essentially a probe of the relatively safety of this environment. These findings are consistent with the results of past research showing the incremental effect of positive emotions over creative performance (Ashby et al., 1999; Forgas, 2000; Isen et al., 1987). Whereas past creativity research induced positive states using different experimental manipulations (Baas et al., 2008; Mastria et al., 2019), in the present study the manipulation was induced through the exposure to specifically designed VEs. The emotional reactions emerging during the exposure to VEs should be therefore taken into consideration as one of the possible mechanisms explaining the influence of virtual reality on the creative behavior. Emotions should thus be included in a multivariate analysis of the creative behavior in VEs, in the attempt to understand whether and to which extent the different characteristic of a VE can impact on different dimensions (i.e., cognition, emotion, personality, motivation, etc.) subsuming the user's creative behavior.

A further interesting result emerging from the current study showed that the Openness personality trait is able to influence participants' creative performance also in VEs. Openness has been indeed showed to be one of the most influential variables affective creative behavior (Corazza \& Agnoli, 2019; Feist, 1998). Even if its effect emerged as consistent in all the tested environments, 
our results revealed that Openness was more effective in increasing response originality in the PVE in comparison to the other environments. This finding seems to indicate that the beneficial effect of the Openness trait is amplified by the characteristics of the virtual environment. This is in line with the hypothesis by Bourgeois-Bougrine et al. (2019) that the atmosphere of the VE could interact with users' attitudes and in particular with their Openness personality trait, producing more original contents. Testing this hypothesis, we demonstrated that the positive atmosphere generated through the PVE interacted with users' Openness trait, which specifically predicted response originality in this environment.

\section{LIMITATIONS AND FUTURE DEVELOPMENTS}

This work is not without limitations. First of all, it would be particularly important replying the effects emerging in this preliminary study using a larger sample of participants. Even if we used a within-subject controlled situation, future studies are required to test and confirm these effects on different types of users (diversified by ages, knowledge and working domains, etc.). Moreover, in the present study we did not design virtual contexts that permitted a complete control and balance of the emotional dimensions. No measures of the valence and arousal dimensions of the cues included in the environments have been performed, which does not allow understanding which specific affective dimension was actually effective in influencing users' creative performance. The design and use of pretested scenarios in terms of valence and arousal could allow future studies to have a higher experimental control in order to clarify our results. On the same vein, it would be interesting eliciting discrete emotions through the use of VEs (Felnhofer et al., 2015) in order to explore the effect of single emotions on creative performance in virtual contexts. Finally, we highlight the need to use real control conditions not only associated to the neutral virtual environment but also to the positive and the negative ones, in order to understand whether the effects emerging in the present study are related to the technological medium or to the different experience in the two types of reality.

Concluding, the use of VEs open new experimental possibilities to understand the effect of emotional reactions on creative performance. Virtual reality allows to generate imaginary environments that could hardly be developed in the real world. Through the change of the contextual cues of a virtual environment, for instance, different emotional reactions could be elicited in the users in order to help them to express at their best their creative potential. In sum, a promising future appears for the study of creativity in virtual environments.

\section{References}

Agnoli, S., Franchin, L., Rubaltelli, E., \& Corazza, G.E. (2015). An eye-tracking analysis of irrelevance processing as moderator of openness and creative performance. Creativity Research Journal, 27(2), 125-132.

Agnoli, S., Pittarello, A., Hysenbelli, D., \& Rubaltelli, E. (2015). "Give, but give until it hurts": The modulatory role of trait emotional intelligence on the motivation to help. PloS one, 10(6), e0130704. 
Ashby, F.G., Isen, A.M., \& Turken, A.U. (1999). A neuropsychological theory of positive affect and its influence on cognition. Psychological Review, 106, 529-550.

Baas, M., De Dreu, C.K., \& Nijstad, B.A. (2008). A meta-analysis of 25 years of mood-creativity research: Hedonic tone, activation, or regulatory focus?. Psychological bulletin, 134(6), 779.

Bourgeois-Bougrine, S., Richard, P., Burkhardt, J.M., Frantz, B., \& Lubart, T. (2020). The expression of users' creative potential in virtual and real environments: An exploratory study. Creativity Research Journal, 32(1), 55-65.

Chirico, A., \& Gaggioli, A. (2019). When virtual feels real: comparing emotional responses and presence in virtual and natural environments. Cyberpsychology, Behavior, and Social Networking, 22(3), 220-226.

Chirico, A., Yaden, D.B., Riva, G., \& Gaggioli, A. (2016). The potential of virtual reality for the investigation of awe. Frontiers in psychology, 7, 1766.

Corazza, G.E. (2016). Potential originality and effectiveness: The dynamic definition of creativity. Creativity Research Journal, 26, 258-267.

Corazza, G.E., \& Agnoli, S. (2020). Personality: Openness. In M. Runco \& S. Pritzker (Eds.), Encyclopedia of Creativity, 3rd Edition. Oxford, UK: Elsevier.

Corazza, G.E., \& Lubart. T., (2021). Intelligence and Creativity: Mapping Constructs on the Space-Time Continuum. Journal of Intelligence, 9: 1. https://doi.org/10.3390/jintelligence9010001

Costa, P.T., \& McCrae, R.R. (1992). Normal personality assessment in clinical practice: The NEO Personality Inventory. Psychological assessment, 4(1), 5.

Faul, F., Erdfelder, E., Lang, A.G., \& Buchner, A. (2007). G* Power 3: A flexible statistical power analysis program for the social, behavioral, and biomedical sciences. Behavior research methods, 39(2), 175-191.

Felnhofer, A., Kothgassner, O.D., Schmidt, M., Heinzle, A.K., Beutl, L., Hlavacs, H., \& Kryspin-Exner, I. (2015). Is virtual reality emotionally arousing? Investigating five emotion inducing virtual park scenarios. International journal of human-computer studies, 82, 48-56.

Feist, G.J. (1998). A meta-analysis of personality in scientific and artistic creativity. Personality and Social Psychology Review, 2(4), 290-309.

Forgas, J.P. (2000). Feeling and thinking: The role of affect in social cognition. Paris: Cambridge University Press.

Goritz A.S., \& Moser K. (2003). Mood and flexibility in categorization: A conceptual replication. Perceptual and Motor Skills, 97(1), 107-119.

Guegan, J., Buisine, S., Mantelet, F., Maranzana, N., \& Segonds, F. (2016). Avatar-mediated creativity: When embodying inventors makes engineers more creative. Computers in Human Behavior, 61, 165-175. https://doi.org/10.1016/j.chb.2016.03.024

Guegan, J., Nelson, J., \& Lubart, T. (2017). The relationship between contextual cues in virtual environments and creative processes. Cyberpsychology, Behavior, and Social Networking, 20(3), 202-206.

Isen A.M., Daubman K.A., \& Nowicki G.P. (1987). Positive affect facilitates creative problem solving. Journal of Personality and Social Psychology, 52(6), 1122-1131.

Jauk, E., Benedek, M., \& Neubauer, A.C. (2014). The road to creative achievement: a latent variable model of ability and personality predictors. European Journal of Personality, 28(1), 95-105.

Kalantari, S., Rounds, J.D., Kan, J., Tripathi, V., \& Cruz-Garza, J.G. (2021). Comparing physiological responses during cognitive tests in virtual environments vs. in identical real-world environments. Scientific Reports, 11(1), 1-14.

Mastria, S., Agnoli, S., \& Corazza, G.E. (2019). How does emotion influence the creativity evaluation of exogenous alternative ideas?. PloS one, 14(7), e0219298.

Michinov, N., Jamet, E., Métayer, N., \& Le Hénaff, B. (2015). The eyes of creativity: Impact of social comparison and individual creativity on performance and attention to others' ideas during electronic brainstorming. Computers in Human Behavior, 42, 57-67. 
Pena, J., Hancock, J., \& Merola, N. (2009). The priming effects of avatars in virtual settings. Communication Research, 36, 838-856.

Ragan, E.D., Bowman, D.A., \& Huber, K.J. (2012). Supporting cognitive processing with spatial information presentations in virtual environments. Virtual Reality, 16(4), 301-314.

Riva, G., Mantovani, F., Capideville, C.S., Preziosa, A., Morganti, F., Villani, D., ... \& Alcañiz, M. (2007). Affective interactions using virtual reality: the link between presence and emotions. Cyberpsychology \& behavior, 10(1), 45-56.

Shin, D. (2018). Empathy and embodied experience in virtual environment: To what extent can virtual reality stimulate empathy and embodied experience?. Computers in Human Behavior, 78, 64-73.

Silvia, P.J., Winterstein, B.P., Willse, J.T., Barona, C.M., Cram, J.T., Hess, K.I., Martinez, J.L., \& Richard, C.A. (2008). Assessing creativity with divergent thinking tasks: exploring the reliability and validity of new subjective scoring methods. Psychology of Aesthtetics Creativity and the Arts, 2, 68-85.

Thornhill-Miller, B., \& Dupont, J.-M. (2016). Virtual reality and the enhancement of creativity and innovation: Under recognized potential among converging technologies? Journal of Cognitive Education and Psychology, 15(1), 102-121.

Vergara, D., Lorenzo, M., \& Rubio, M.P. (2017). Virtual environments in materials science and engineering: the students' opinion. In Materials Science and Engineering: Concepts, Methodologies, Tools, and Applications (pp. 1465-1483). IGI Global.

Verhaeghen P., Joormann J., \& Khan R. (2005). Why we sing the blues: The relation between self- reflective rumination, mood, and creativity. Emotion, 5, 226-232.

Watson, D., \& Clark, L.A. (1999). The PANAS-X: Manual for the positive and negative affect schedule-Expanded form. University of lowa: Psychology Publications.

Wilson, R.C., Guilford, J.P., \& Christensen, P.R. (1953). The measurement of individual differences in originality. Psychological Bulletin, 50(5), 362.

(c) Copyright by Faculty of Education, University of Bialystok,

20 Swierkowa St., 15-328 Bialystok, Poland

tel. +48857457283

e-mail: creativity@uwb.edu.pl

http://www.creativity.uwb.edu.pl 